\title{
ESTUDO DA EFICÁCIA E TOLERÂNCIA DO ARTESUNATO ORAL ISOLADO E EM ASSOCIAÇÃO COM MEFLOQUINA, NOTRATAMENTO DA MALÁRIA FALCIPARUM NÃO COMPLICADA EM ÁREA ENDÊMICA DO PARÁ, BRASIL
}

\author{
Bernardo da Silva Cardoso, Heitor Vieira Dourado, Maria da Conceição \\ Nascimento Pinheiro, José Angelo Barlleta Crescente, Walter Wanderley \\ Amoras, Jorge Baena e Sandra Saraty
}

\begin{abstract}
Com o objetivo de avaliar a eficácia e tolerância do artesunato no tratamento da malária falciparum não complicada em área endêmica do Estado do Pará, 153 pacientes foram randomizados e estudados em três grupos, distribuidos por esquema terapêutico (I recebeu mefloquina 1000mg; II utilizou artesunato 1000mg; III usou a combinação de $600 \mathrm{mg}$ de artesunato seguida de 500 de mefloquina). A avaliaçào constou de exame clinico e parasitológico diariamente nos primeiros 7 dias $e$ semanalmente até o $35^{\circ}$ dia do acompanbamento e de análise bioquímica e hematológica realizada antes e no $7^{\circ}$ dia, visando o controle de cura e a identificação de possiveis efeitos associados à administração das drogas. Os grupos estudados foram bomogêneos quanto ao sexo, parasitemia e presença de febre. O tempo para desaparecimento da parasitemia foi mais curto nos grupos II e III, respectivamente, cujos esquemas terapêuticos empregaram artesunato. O desaparecimento da febre foi mais räpido no grupo tratado com a combinação das drogas. Alteraçôes clinicas e bioquímicas associadas a administração das drogas não mostraram diferenças significativas entre os grupos estudados. O desaparecimento precoce da febre e parasitemia, e a ausência de importantes efeitos indesejáveis, sugerem que artesunato administrado isoladamente ou em combinaçâo com mefloquina constituem medidas terapêuticas capazes de contribuir para o controle da doença na região.
\end{abstract}

Palavras-chaves: Artesunato. Antimalárico. Eficácia. Malária falciparum. Mefloquina.

No Brasil, dentre as doenças transmissíveis, a malária representa o principal problema sanitário na atualidade, sendo que em torno de $98 \%$ dos casos ocorre na Região Amazônica ${ }^{12}$. Dos exames positivos para $P$. falciparum, espécie responsável pelas formas mais graves e por freqüentes recrudescências, 99\% ocorreram nessa região ${ }^{12}$. Infere-se que a interrelação de fatores econômicos, sociais, ambientais, tais como: intensificação do processo de migração interna, desenvolvimento de projetos econômicos em regiôes endêmicas, proliferação de cepas de $P$. falciparum resistente, contribuam para a conseqüente

Núcleo de Medicina Tropical da Universidade Federal do Pará. Fundação Nacional de Saúde.

Apoio financeiro: Laboratório Mepha - Switzerland.

Endereço para correspondência: $\mathrm{Dr}^{2}$ Maria da Conceição $\mathrm{N}$. Pinheiro. Av. Generalíssimo Deodoro 92, Bairro Umarizal, 66055- 240 Belém, PA.

Recebido para publicação em 10/02/95. elevação das taxas de morbidade e mortalidade, nessa região ${ }^{13}$.

Segundo dados do Sistema de Informações sobre Malária, da Fundação Nacional de Saúde, os estados de Rondônia, Mato Grosso e Pará são detentores dos principais focos ativos dessa doença, por apresentarem os maiores números de casos do país ${ }^{13}$.

Nas últimas décadas, esses estados amazônicos têm apresentado os mais elevados indices de crescimento populacional, como reflexo da política de ocupação econômica desenvolvida pelo Governo Federal, a partir dos anos 70 .

Nesse processo, intensos e desorganizados fluxos migratórios rumaram para a Amazônia, formados por trabalhadores expulsos do campo pelo avanço do latifúndio, enquanto outras categorias de trabalhadores fugiam da periferia das grandes cidades evacuados pelo desemprego. Os conhecidos "grandes projetos", 
Cardoso BS, Dourado HV, Pinheiro MCN, Crescente JAB, Amoras WW, Baena J. Saratu S. Estudo da eficácia e tolerância do artesunato oral isolado e em associação com mefloquina. no tratamento da malária falciparum não complicada em ârea endêmica do Pará-Brasil. Revista da Sociedade Brasiletra de Medicina Tropical 29:251-257, mai-jun, 1996.

como as construções de rodovias, hidrelétricas, exploração mineral (mineração e garimpagem), projetos de colonização, dentre outros, funcionavam como frentes fetichistas de atração, na busca da sobrevivência ${ }^{2}$

Nesse contexto, o perfil epidemiológico da Amazônia brasileira foi célebre e drasticamente alterado, ao sofrer profundas transformaçōes ambientais, nos seus aspectos físicos, biológicos e sociais, que afetaram o meio ambiente e tornaram péssimas as já precárias condiçôes de vida e de trabalho da maioria da população dessa região².

A resistência do $P$. falciparum nessa região a certos fármacos específicos, tais como cloroquina, amodiaquina, quinino e sulfas, dentre outros, tem sido registrada ${ }^{5}{ }^{14}$. Essa questão vem contribuindo para o uso de tratamentos mais onerosos e menos eficazes, que ainda parecem aumentar os níveis de risco da intolerância e de intoxicações, além de manter ativos focos de irradiação da malária.

$\mathrm{Na}$ maior parte dos países endêmicos, O objetivo da luta contra o paludismo é evitar a mortalidade e reduzir a morbidade e as perdas sócio-econômicas ocasionadas pela enfermidade $^{11}$. O uso de drogas antimaláricas, nos últimos tempos, tem sido uma das opções viáveis na busca do controle dessa doença. A mefloquina, por exemplo, vem sendo empregada em diferentes áreas endêmicas do mundo, visando o tratamento da malária, por $P$. falciparum, resistente à cloroquina ${ }^{34} 10161920$. Estudos recentes sobre a resistência in vitro do $P$. falciparum a antimaláricos, em áreas endêmicas da Amazônia, não evidenciaram cepas resistentes à mefloquina ${ }^{56}$.

$O$ artesunato, um derivado da artemisinina, utilizado mais recentemente no tratamento da malária pelo P. falciparum, tem-se mostrado efetivo no rápido clareamento da parasitemia. Entretanto, tem sido também observado índices de recrudescência, que variam de 4,9 a $56 \% \%^{151821}$

A combinação de drogas que atuam no rápido clareamento da parasitemia, como o artesunato a outra de ação residual com a mefloquina, foi usada por Looareensuwan et al na Tailândia, apresentando-se altamente eficaz e bem tolerada em pacientes com malária falciparum não complicada ${ }^{9}$.

- Visando contribuir na luta contra malária, na Região Amazônica, este estudo tem como objetivo avaliar a eficácia e a tolerância do artesunato isolado e associado à mefloquina, no tratamento da malária falciparum, não complicada na região garimpeira de Itaituba onde são registrados elevados índices dessa doença $a^{13}$.

\section{MATERIAL E MÉTODOS}

Sede da pesquisa. O estudo foi desenvolvido no período de agosto de 1993 a dezembro de 1994, na Unidade Mista Itaituba, estabelecimento hospitalar da Fundação Nacional de Saúde (FNS) localizado na sede do município de Itaituba, há $800 \mathrm{~km}$ da capital do Estado do Pará. Essa região caracteriza-se epidemiológicamente como área de alta transmissão de malária ${ }^{13}$, onde a resistência do $P$. falciparum à cloroquina e ao quinino tem sido registrada ${ }^{56}$.

Seleção dos pacientes. Foram aceitos para o estudo pacientes apresentando: a) sinais e sintomas de malária não complicada; b) parasitemia assexuada positiva de $P$. falciparum; c) idade variando de 14 a 60 anos; d) consentimento por escrito para participar do estudo.

Não participaram: a) portadores de malária falciparum impossibilitados de permanecer na sede do município (fora da área de transmissão) durante o período básico de observação de 35 dias; b) malária mista ; c) processo infeccioso grave associado; d) menores de 12 anos; e) gestantes; f) malária concominante com doenças degenerativas graves (cardiopatias, hepatopatias, nefropatias, etc); g) uso prévio de antimalárico nos 15 dias precedentes ao estudo.

Constituição dos grupos. A região aurífera do Tapajós foi considerada como universo da pesquisa, enquanto a demanda da Unidade Mista de Itaituba (UMI) representou a população alvo, sendo que a população da amostra adotada foi a do conjunto de casos de malária dessa demanda. A unidade de estudo considerada foi cada paciente com malária falciparum, obedecendo os critérios de inclusão no estudo. O tipo de amostragem foi probabilística casual simples sem reposição, em que os casos foram distribuídos em três grupos (I, II, III), cada grupo com número igual de casos, correspondendo a um tipo de esquema terapêutico. A técnica ultilizada para a determinação do esquema terapêutico a ser 
Cardoso BS, Dourado HV, Pinheiro MCN, Crescente JAB, Amoras WW, Baena J, Saraty S. Estudo da eficácia e tolerância do artesunato oral isolado e em associação com mefloquina, no tratamento da malária falciparum não complicada em área endêmica do Pará - Brasil. Revista da Sociedade Brasileira de Medicina Tropical 29:251-257, mai-jun, 1996.

aplicado em cada paciente foi a do sorteio dos três primeiros, que serviram de referência para o processo seqüencial de admissão e respectiva terapia.

\section{Esquemas terapêuticos.}

Grupo I. Mefloquina lactab (250mg). Dose total $1000 \mathrm{mg}$.

Administração via oral em duas tomadas de $500 \mathrm{mg}$ com intervalo de 8 horas.

Grupo II. Artesunato lactab (50mg). Dose total $1000 \mathrm{mg}$. Administração via oral:

1 dia: $200 \mathrm{mg}$ de 12 em 12 horas

$2^{\circ}$ dia: $100 \mathrm{mg}$ de 12 em 12 horas

$3^{\circ}$ dia: $100 \mathrm{mg}$ de 12 em 12 horas

$4^{\circ}$ dia: $100 \mathrm{mg}$ de $12 \mathrm{em} 12$ horas

Grupo III. Artesunato Lactab (50mg). Dose total - 600mg:

$1^{\circ}$ dia: $200 \mathrm{mg}$ de 12 em 12 horas

$2^{\circ}$ dia: $200 \mathrm{mg}$.

Mefloquina lactab (250mg). Dose total $500 \mathrm{mg}$. Dose única, 12 horas após a última dose de artesunato.

Para os casos de falha do tratamento (recrudescência), foi prevista a utilização da mefloquina - $16,5 \mathrm{mg} / \mathrm{kg}$ de peso, divididas em duas tomadas, com intervalo de 8 horas entre as doses.

Investigação clínico-laboratorial. Todos os pacientes foram admitidos para tratamento hospitalar. Antes do tratamento, foram registrados em formulário protocolar dados sobre a história clínica, sinais físicos (incluindo temperatura axilar, pulso e pressão arterial), contagem quantitativa de parasitas no sangue periférico, e testes hematológicos e bioquímicos (hemograma, bilirrubinas total e fraçôes, aminotransferases, fosfatase alcalina, creatinina). Com exceção dos testes hematológicos e bioquímicos, que só foram repetidos sete dias após o início do tratamento, os demais procedimentos foram reavaliados diariamente nos primeiros sete dias e, posteriormente, no $14^{\circ}, 21^{\circ}, 28^{\circ}$ e $35^{\circ}$ dias, a contar do inicio da terapêutica. A aferição da temperatura axilar foi feita de 12 em 12 horas e a parasitemia quantitativa de $24 \mathrm{em} 24$ horas.

O diagnóstico de malária foi confirmado através do exame ao microscópio óptico de uma gota espessa de sangue periférico (punção digital) em lâmina corada pelo Giemsa. Foram contadas formas assexuadas de $P$. falciparum em 100 campos microscópicos e a densidade parasitária foi expressa em parasitas por $\mathrm{mm}^{3}$ de sangue.

As condições do paciente determinavam a oportunidade de alta hospitalar, após caracterizados os procedimentos essenciais ao acompanhamento dos casos que prosseguiam de forma ambulatorial. Essas medidas visavam - controle de cura (ausência de formas assexuadas de $P$. falciparum no sangue periférico após o tratamento e durante $\mathrm{O}$ acompanhamento de 35 dias), e a avaliação da tolerância que foi baseada na evolução clínica e laboratorial.

Avaliação dos resultados (Análise estatística). O tamanho da amostra em cada grupo permite detectar uma diferença de $10 \%$ nas medidas de eficácia (clearence da parasitemia, desaparecimento da febre) entre os grupos, a um nível de confiança de $95 \%$. Os dados foram sumarizados por medidas de posição e dispersão não-paramétricas (mediana e amplitude) ou paramétricas (média e desviopadrão), quando oportunos. A independência entre frequiências foi avaliada pelo teste do qui-quadrado, com correção de continuidade. A comparação entre as distribuições dos valores da parasitemia, duração da febre/parasitemia, idade e parâmetros bioquímicos foi feita pelo teste de MannWhitney (dois grupos) ou de Kruskal-Wallis (três grupos). Em todos os casos, foi estabelecido 95\% como nível de confiança para inferência estatística.

\section{RESULTADOS}

Dentre 153 pacientes com malária falciparum inscritos para o estudo da eficácia e tolerância ao artesunato, 133 foram acompanhados durante os 35 dias estabelecidos para o contrôle. Os doentes apresentavam manifestações clínicas e parasitológicas de malária falciparum não complicada, tendo o sexo masculino contribuído com a maioria dos casos. A menor idade foi observada no grupo tratado com a combinação artesunato + mefloquina. Estatísticamente, os grupos estudados mostraram-se homogêneos quanto ao sexo, intensidade da parasitemia e presença de febre (Tabela 1)

Dezenove pacientes abandonaram o contrôle, sendo 5, 5 e 9 pertencentes aos grupos 
Cardoso BS, Dourado HV, Pinbeiro MCN, Crescente JAB, Amoras WW, Baena J, Saraty S. Estudo da eficácia e tolerância do artesunato oral isolado e em associação com mefloquina, no tratamento da malária falciparum não complicada em ärea endêmica do Pará - Brasil. Revista da Sociedade Brasileira de Medicina Tropical 29:251-257, mai-jun, 1996.

Tabela 1 - Caracteristicas dos grupos quanto a sexo. idade, parasitemia e presença de febre antes da terapeutica

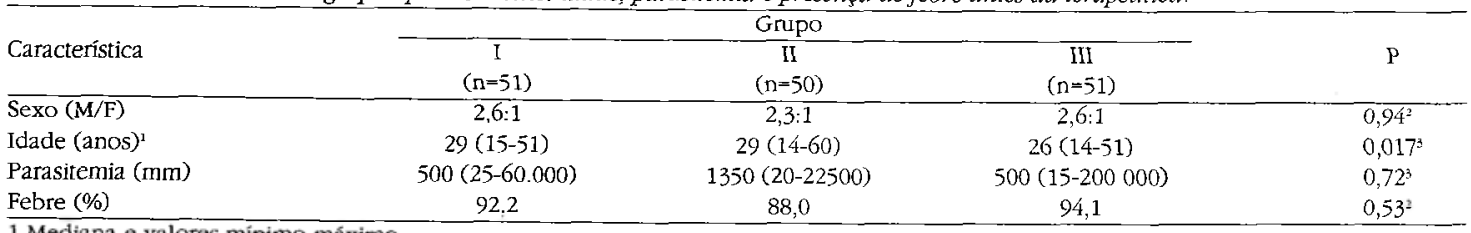

1 Mediana e valores mínimo-máximo

2 Teste do Qui-quadrado com correção de continuidade

3 Teste de Kruskal-Wallis

I, II e III, respectivamente. Não houve diferença significativa quanto ao sexo, parasitemia e febre entre o grupo tratado e aquele que abandonou o estudo (Tabela 2).

Tabela 2 - Comparação entre o grupo tratado que permaneceu $e$ aquele que abandonou o controle de 35 dias.

\begin{tabular}{|c|c|c|c|}
\hline \multirow[b]{2}{*}{ Caracteristica } & \multicolumn{2}{|c|}{ Grupo } & \multirow[b]{2}{*}{$\mathrm{P}$} \\
\hline & tratados & abandono & \\
\hline $\operatorname{Sexo}(M / F)$ & $2,6: 1$ & $2,2: 1$ & $0,94^{2}$ \\
\hline Idade $(a n o s)^{1}$ & $29(14-60)$ & $23(14-35)$ & $0,002^{3}$ \\
\hline Parasitemia $^{1}(\mathrm{~mm})$ & $500(15-200000)$ & $2500(15-60000)$ & $0,065^{3}$ \\
\hline Febre (\%) & 78,9 & 93,2 & $0,081^{2}$ \\
\hline
\end{tabular}

Um paciente do grupo II apresentou forma mista da doença no segundo dia após o início da terapêutica, tendo sido excluído do estudo e medicado com primaquina.

O tempo para o desaparecimento da parasitemia foi menor no grupo II e III, 25,7 \pm 6,3 e 26,4 $\pm 7,3$ horas (média desvio padrão) respectivamente, cujos esquemas terapêuticos empregavam artesunato isolado e em associação com mefloquina. O desaparecimento da febre foi mais rápido no grupo III $(\mathrm{P}=0,019)$ (Tabela 3).
Dentre os pacientes avaliados até o $35^{\circ}$ dia, a cura ocorreu em 100\% nos grupos tratados com mefloquina e artesunato isoladamente, $\mathrm{e}$ $95,2 \%$ quando tratado com a combinação das drogas. A falência terapêutica ao esquema III foi registrada entre o $7^{\circ}$ e $14^{\circ}$ dia no primeiro caso e entre o $14^{\circ}$ e $21^{\circ}$ dia no segundo caso.

Dez foram os efeitos colaterais observados nos três grupos. Dentre eles, tontura foi o de maior freqüência entre os pacientes tratados com mefloquina isolada $(P=0,0010)$ Estatísticamente, a ocorrência dos demais efeitos colaterais não se diferencia entre os grupos tratados (Tabela 4).

Tabela 4 - Efeitos colaterais associados aos esquemas terapêuticos usados pelos pacientes do gripo I, II e III.

\begin{tabular}{|c|c|c|c|c|c|c|}
\hline \multirow[b]{2}{*}{ Efeitos colaterais } & \multicolumn{5}{|c|}{ Grupo } & \multirow[b]{2}{*}{$P$} \\
\hline & \multicolumn{2}{|c|}{$\begin{array}{c}I \\
(n=46)\end{array}$} & II & $\begin{array}{l}\text { II } \\
-45)\end{array}$ & $\begin{array}{c}\text { III } \\
(n=42)\end{array}$ & \\
\hline Tontura & 14 & 304 & 1 & 23 & 6143 & 00010 \\
\hline Fraqueza & & 196 & 4 & 8.9 & 5119 & 0.30 \\
\hline Naúseas & 8 & 17,4 & 4 & 8,9 & $2 \quad 4,8$ & 0,14 \\
\hline Cefaléia & 2 & 4,3 & 4 & 8,9 & 716,7 & 0,14 \\
\hline Diarreia & 4 & 8,7 & 5 & 11,1 & 37,1 & 0,80 \\
\hline Vômitos & 4 & 8,7 & 4 & 8,9 & 2,4 & 0,39 \\
\hline Dor abdominal & 2 & 4,3 & 5 & 11,1 & 4.8 & 0,36 \\
\hline Artralgia & 3 & 6,5 & - & - & 9,5 & 0,12 \\
\hline Ictericia & - & - & - & - & 4,8 & - \\
\hline Tïnitus & 1 & 2,2 & - & - & - & - \\
\hline Algum efeito & 24 & 52,2 & 14 & 56,0 & $17 \quad 32,7$ & 0,12 \\
\hline
\end{tabular}

1 Teste do Qui-quadrado com correção de continuidade

Tabela 3 - Resposta terapêutica: desaparecimento da febre e parasitemia no periodo de 35 dias.

\begin{tabular}{|c|c|c|c|c|}
\hline Característica & $\begin{array}{c}I \\
(n=51)\end{array}$ & $\begin{array}{c}\text { II } \\
(n=50)\end{array}$ & $\begin{array}{c}\text { III } \\
(n=51)\end{array}$ & $\mathbf{P}$ \\
\hline \multicolumn{5}{|c|}{ Resultado do tratamento } \\
\hline cura & 46 & 45 & 40 & $0,14^{1}$ \\
\hline recrudescência & - & - & 2 & - \\
\hline abandono & 5 & 5 & 9 & 0,39 \\
\hline \multicolumn{5}{|c|}{ Tempo de desaparecimento $(h)^{\circ}$} \\
\hline parasitemia & $32,4 \pm 14,7$ & $25,7 \pm 6,3$ & $26,4 \pm 7,3$ & 0,0133 \\
\hline febre & $28,5 \pm 10,8$ & $28,1 \pm 9,1$ & $25,2 \pm 5,3$ & 0,0193 \\
\hline
\end{tabular}

1 Mediana e valores mínimo-maximo

2 Teste do Qui-quadrado com correção de continuidade

3 Teste de Kruskal-Wallis. 
Cardoso BS, Dourado HV, Pinheiro MCN, Crescente JAB, Amoras WW, Baena J, Saraty S. Estudo da eficácia $e$ tolerância do artesunato oral isolado e em associação com mefloquina, no tratamento da malâria falciparum não complicada em área endêmica do Pará - Brasil. Revista da Sociedade Brasileira de Medicina Tropical 29:251-257, mai-jun, 1996

Observa-se diferença estatística nos níveis de bilirrubina conjugada, sendo significativamente menor no grupo de pacientes tratados com esquema II (artesunato isoladamente). Variações não importantes nos demais parâmetros. Discreta redução nos níveis de hemoglobina e aumento da alanina aminotransferase (ALT) no grupo II

\section{DISCUSSÃO}

Ensaios clínicos com derivados da artemisinina (artemether, artesunato) em diferentes regimes terapêuticos têm sido testados no tratamento da malária falciparum não complicada, resultando em desaparecimento precoce da parasitemia e registros de taxas variáveis de recrudescência $(4,97 \text { a } 50 \%)^{101521}$.

$\mathrm{Na}$ Amazônia brasileira, onde concentramse $98 \%$ dos casos de malária do país ${ }^{12}{ }^{13}$, resistência à cloroquina e outros antimaláricos tradicionais tem sido registrada ${ }^{5614}$. O emprego do artesunato oral, em regime de $50 \mathrm{mg}$ a cada 12 horas durante 6 dias, evidenciou a possibilidade de curar $95 \%$ de um grupo de pacientes com malária falciparum em área de grande transmissão dessa região ${ }^{21}$.

$\mathrm{Na}$ Tailândia foi observada uma freqüência maior de recrudescência, quando se empregava artemisinina oral em regime de tratamento menor que cinco dias ${ }^{15}$.

Na série de casos tratados com artesunato isoladamente deste estudo, foi alcançada uma relação de $100 \%$ de cura, empregando-se dose total de $1000 \mathrm{mg}$ em regime de quatro dias. Looareesuwan et al observaram $88 \%$ de cura utilizando $50 \mathrm{mg}$ a cada 12 horas durante 5 dias (600mg dose total) ${ }^{10}$.

Segundo a Organização Mundial de Saúde (OMS $)^{17}$, quando as artemisininas se combinam com mefloquina em tratamento de 3 dias, o recrudescimento dos sintomas, se reduzem a $3 \%$. Essa combinação tem sido extensivamente estudada em regimes de tratamento variando de 3 a 6 dias, mostrando resultados promissores $^{40}$.

Alecrin et al, utilizando derivado de artemisinina durante 5 dias com mefloquina no $3^{\circ}$ dia do tratamento da malária falciparum, em área de transmissão da Amazônia, não evidenciaram recrudescência na avaliação de 35 dias de acompanhamento ${ }^{1}$.

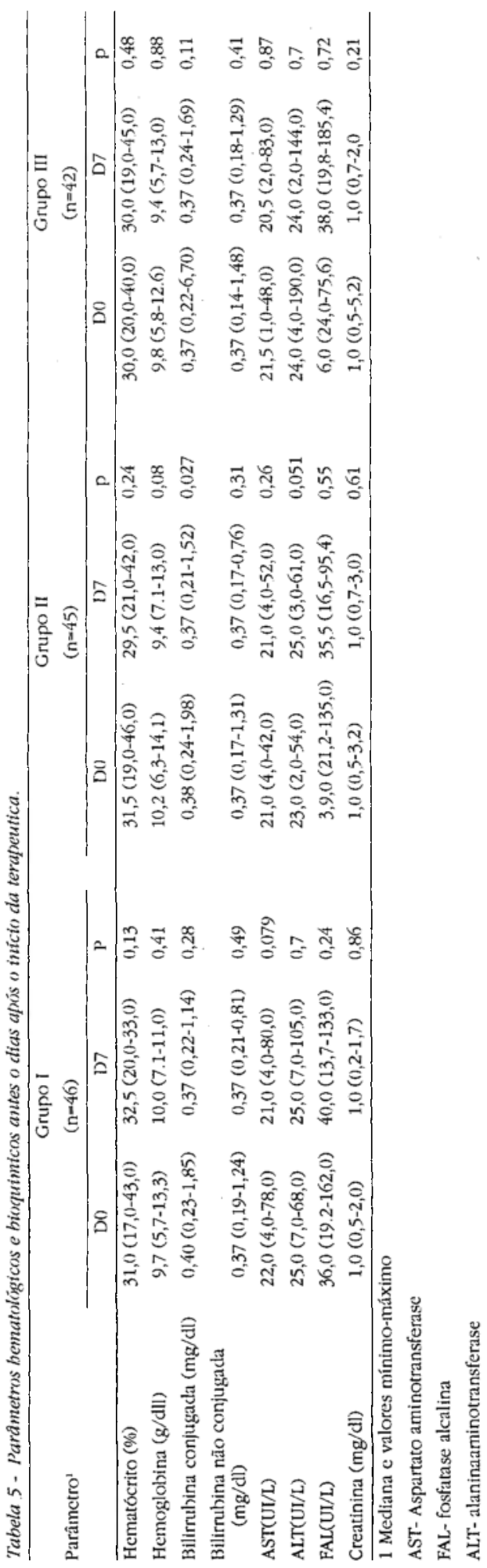


Cardoso BS, Dourado HV, Pinbeiro MCN, Crescente JAB, Amoras WW, Baena J, Saraty S. Estudo da eficácia e tolerância do artesunato oral isolado e em associação com mefloquina, no tratamento da malária falciparum não complicada em área endêmica do Pará-Brasil. Revista da Sociedade Brasileira de Medicina Tropical 29:251-257, mai-jun, 1996.

Diferente dos resultados de outros estudos $^{110}$ em que a combinação de drogas curou $100 \%$ dos casos de malária não complicada, na série do presente estudo houve falha do tratamento em dois casos, sendo registrada a partir do reaparecimento da parasitemia em D14 e D21 no $1^{\circ}$ e $2^{\circ}$ casos respectivamente, provavelmente devido à recrudescência do P. falciparum, considerandose que todos os pacientes foram mantidos durante os 35 dias de contrôle fora da área de transmissão.

O desaparecimento da parasitemia nos grupos tratados com artesunato ocorreu dentro de $25,7 \pm 6,3$ e $26,4 \pm 7,3 \mathrm{~h}$ no grupo II e III, respectivamente. Nos estudos de Looareensuwan et al, na Tailândia, utilizando dose total de $600 \mathrm{mg}$ de artesunato, o tempo para o desaparecimento da parasitemia foi maior do que o observado neste estudo.

A combinação das drogas foi usada visando a redução precoce da parasitemia com artesunato $\mathrm{e}$ a clareamento residual com mefloquina, desta forma contribuindo para a diminuição da fonte de infecção e transmissibilidade da doença.

As manifestações clínicas associadas ao uso das drogas (efeitos colaterais) foram leves e regrediram logo após o término do tratamento, não havendo necessidade de interromper o esquema. A freqüência de tontura foi significativamente maior no grupo tratado com mefloquina isolada. Este sintoma tem sido freqüente em outros estudos empregando mefloquina ${ }^{10}$.

Parâmetros hematológicos e bioquímicos não mostraram alterações significativas após a terapêutica empregada, a maioria dos valores bioquímicos se mantiveram dentro dos padrões de normalidade. No estudo de Looareenswan et al 1992, também não foram identificadas alterações importantes nos testes laboratorias de pacientes tratados com artesunato isolado ou associado à mefloquina.

O desaparecimento precoce da febre e da parasitemia, a ausência de importantes efeitos indesejáveis, sugerem que o artesunato administrado isoladamente ou em combinação com mefloquina constituem medidas terapêuticas que podem contribuir para o controle da doença na região.

\section{SUMMARY}

With the objective to evaluate the efficacy and tolerance of artesunate in the treatment of noncomplicated falciparum malaria in endemic area of the State of Pará, 153 patients were randomized and studied in three groups, distributed by therapeutical scheme (I received mefloquine $1000 \mathrm{mg}$, II used artesunate 600mg followed by mefloquine $500 \mathrm{mg}$ ). Evaluation was made by daily clinical and parasitbological examination, in the first 7 days, and weekly until the 35th day of the follow up. Biochemical and bematological analysis previously done and on the 7 th day, targetting cure control and identification of possible effects related to drugs administration. As to sex, parasitemy and fever, studied groups were homogeneous. Time for parasitemy disappearence was shorter in the groups II and III respectively, whose therapeutical schemes bad artesunate. Fever disappereance was quicker in the group treated with the combination of drugs Clinical and biochemical alterations associated with drugs administration did not show significant differences among the studied groups. Early disappearence of fever and parasitemy, and absence of important side effects suggest that artesunate, isolated or administrated in combination with mefloquine, constitutes an able therapeutical procedure to contribute for disease control in that region.

Key-words: Artesunate. Anti-malaric. Efficacy. Falciparum malaria. Mefloquine.

\section{REFERÊNCIAS BIBLIOGRÁFICAS}

1. Alecrin WD, Silva Junior RC, Chaves AGS, Chalub LMC, Alecrin MGC, Silva EB. Tratamento da malária Plasmodium falciparum com a associação de artemether mais mefloquina. $I n$ : Resumos do XXXI Congresso da Sociedade Brasileira de Medicina Tropical, São Paulo p. 160, 1995.

2. Amoras WW. A garimpagem na Amazônia: doenças, desordem e descaso. Uma visão do garimpo do Crepari (PA). Tese de mestrado, Universidade Federal do Pará, Belém, PA, 1991.

3. Bhatt KM. Mephaquin (mefloquina) in treatment of clroloquine resistant falciparum malaria patients in Nairabi, Kenia. In: Abstract of XIII International Congress for Tropical Medicine and Malaria, Jomtien, 1992.

4. Bunnag D, Kanda T, Karbwang $\mathrm{Y}$, Thimasarn $\mathrm{K}$, Pingpak S, Harinasuta T. Artemether-mefloquine 
Cardoso BS, Dourado HV, Pinbeiro MCN, Crescente JAB, Amoras WW, Baena J, Saraty S. Estudo da eficácia $e$ tolerância do artesunato oral isolado e em associação com mefloquina, no tratamento da malária falciparum não complicada em área endêmica do Pará - Brasil. Revista da Sociedade Brasileira de Medicina Tropical 29:251-257, mai-jun, 1996.

combination in multidrug resistant falciparum malaria. Transactions of the Royal Society of Tropical Medicine and Hygiene 89:213-215, 1995.

5. Couto AA, Calvosa VS, Lima JE, Souza JM. Evolução da resistência in vitro das cepas do Plasmodium falciparum a antimaláricos em áreas de prospecção de ouro no Estado do Amapá entre 1983 e 1990. Revista da Sociedade Brasileira de Medicina Tropical 26:215-220, 1993.

6. Couto AA, Calvosa VS, Santos MA, Souza JM. Resistência in vitro de cepas do Plasmodium falciparum isolados no sul do Estado do Pará, em diferentes períodos: emergência de casos de multiresistência. Revista da Sociedade Brasileira de Medicina Tropical 26:5-9, 1993.

7. Dourado HV, Abdon NP, Martins SJ. Falciparum malaria epidemiology in latin America, biologia and clinical considerations, treatment and prophylaxis. Infectious Disease Clinics of North America 8:207-223, 1994.

8. Lachi J. M. Introduction to sample Size Determination and Power Analysis for Clinical trials control. Clin. Trials 2:93-113,1981.

9. Looareensuwan S, Kyle DE, Viravan C, Vanijanonta S, Wilairatana P, Charoenlarp P, Canfield CJ, Webster HK. Treatment of patient with recrudescent falciparum malaria with a sequential combination of artesunato and mefloquine. The American Journal of Tropical Medicine and Hygiene 47:794-799, 1992.

10. Looareensuwan $S$, Viravan $C$, Vanijanonta $S$, Wilairatana P, Suntharasamai $P$, Charoenlarp $P$, Arnold K, Kyle DE, Canfield CJ, Webster HK, Randomised trial of artesunate and mefloquine alone and in sequence for acute uncomplicate falciparum malaria. The Lancet 339:821-824, 1992.

11. Organizacion Mundial de la Salud. La Lucha mundial al paludismo. Boletin de la Oficina Sanitaria Panamericana 116:477-482, 1994.

12. Marques A. Dados Epidemiológicos de malária na Amazônia por município, referente a 1992. Revista Brasileira de Medicina Tropical 26:43-59, 1993.
13. Marques AC, Gitierrez HC. Combate a malária no Brasil: evolução, situação atual e perpectivas. Revista da Sociedade Brasileira de Medicina tropical 27 (supl):91-108, 1994.

14. Neifer S, Kremsner PG. Drug susceptibility of Plasmodium falciparum in the western Amazon Region State of Acre, Brazil. Revista do Instituto de Medicina Tropical de São Paulo 33:205-211, 1991.

15.Nguyeu Duy SY. Administración oral de artemisina contra la malaria. Boletin de la Oficina Sanitaria Panamericana 115:342-343, 1993.

16.Niangué $\mathrm{J}$. Clinical study of Mephaquin (Mefloquine) in the treatment of chloroquineresistant falciparum malaria in Abdjan, Ivory Coas. In: Abstract of XIII International Congress for'Tropical Medicine and Malaria, Jomtien, 1992.

17. Organización Mundial de la Salud. Chinese herb remedy beats multi-drug resistant malaria. Feature No. 175, abril de 1994.

18. Silva RSU, Souza JM, Pinto AYN, Calvosa VSP, Abdon NP, Gonçalves ER, Viana RM. Papel do artesunato em comprimidos no trabalho da FNS$\mathrm{CR}$ do Pará, 1993. In: XXX Congresso da Sociedade Brasileira de Medicina Tropical, Salvador, p. 271, 1994.

19. Some- Ekobe A, Somo-Moyou R, Lohoue J. Efficacy and tolerance of mephaquin in a malaria-endemic zone. In: Abstract of XIII International Congress for Tropical Medicine and Malaria, Jomtiem, 1992.

20. Than M, Tha KK, Aye SS, Soe AY, Win K. Multidrug resistant falciparum malaria treated with mefloquine and sodium artesunate. In: Abstract of XI International Congress for Tropical Medicine and Malaria, Thailand, 1992.

21. Uchôa R, Coelho PKP, Miranda R, Pereira J, Lopes J, Storch M, Souza JM. Artemisinina em esquemas alternativos no Tratamento da malária falciparum, Marabá-PA. In: Resumo do XXXI Congresso da Sociedade Brasileira de Medicina Tropical, São Paulo p. 160, 1995. 